\title{
Replicable Services for Reproducible Research: A Model for Academic Libraries
}

\author{
Franklin Sayre and Amy Riegelman
}

Over the past decade, evidence from disciplines ranging from biology to economics has suggested that many scientific studies may not be reproducible. This has led to declarations in both the scientific and lay press that science is experiencing a "reproducibility crisis" and that this crisis has consequences for the extent to which students, faculty, and the public at large can trust research. Faculty build on these results with their own research, and students and the public use these results for everything from patient care to public policy. To build a model for how academic libraries can support reproducible research, the authors conducted a review of major guidelines from funders, publishers, and professional societies. Specific recommendations were extracted from guidelines and compared with existing academic library services and librarian expertise. The authors believe this review shows that many of the recommendations for improving reproducibility are core areas of academic librarianship, including data management, scholarly communication, and methodological support for systematic reviews and data-intensive research. By increasing our knowledge of disciplinary, journal, funder, and society perspectives on reproducibility, and reframing existing librarian expertise and services, academic librarians will be well positioned to be leaders in supporting reproducible research.

\section{Introduction}

In recent years, evidence has emerged from a number of disciplines, including psychology, ${ }^{1}$ biology, ${ }^{2}$ biomedicine, ${ }^{3}$ neuroscience, ${ }^{4}$ drug development, ${ }^{5}$ chemistry, ${ }^{6}$ climate science, ${ }^{7}$ economics, ${ }^{8}$ and education ${ }^{9}$ that science may be facing a reproducibility crisis. The authors have argued previously that libraries have a responsibility to help support reproducible research and should take a leadership role within academic institutions. ${ }^{10}$ This paper outlines a model for how academic libraries can broadly support reproducible research based on the alignment between recommendations contained in guidelines developed by funders, professional societies, and publishers for improving reproducibility and existing expertise and services offered by academic libraries.

As the authors explained in a previous article, the terms reproducibility and replicability are often misused, and it is important to understand the differences. ${ }^{11}$ The previously published editorial contains a longer discussion of issues with current definitions and briefly outlines

Franklin Sayre is a Liaison Librarian at the University of Minnesota Twin Cities and Amy Riegelman is a Social Sciences Librarian at the University of Minnesota Twin Cities; email: franklin.sayre@gmail.com, aspringe@umn.edu. 
the most useful distinctions, ${ }^{12}$ but to summarize, the National Science Foundation's (NSF) Social, Behavioral and Economic (SBE) Division Subcommittee on Replicable Science published a report on robust research practices, where they define reproducibility as "the ability of a researcher to duplicate the results of a prior study using the same materials and procedures [emphasis added] as were used by the original investigator." ${ }^{13}$ Replicability was defined as "the ability of a researcher to duplicate the results of a prior study if the same procedures are followed but new data are collected [emphasis added]." ${ }^{14}$ Leek and Jager reinforce this definition, noting that "[a] study is reproducible if all of the code and data used to generate the numbers and figures in the paper are available and exactly produce the published results." 15

Stodden et al. have proposed a continuum of reproducible research in order to further explore the concept of reproducible research (table 1). ${ }^{16}$

\section{TABLE 1 \\ Levels of Reproducibility*}

- Reviewable Research. The descriptions of the research methods can be independently assessed and the results judged credible. (This includes both traditional peer review and community review and does not necessarily imply reproducibility.)

- Replicable Research. Tools are made available that would allow one to duplicate the results of the research (for example, by running the author's code to produce the plots shown in the publication). Here, tools might be limited in scope (for example, only essential data or executables) and might only be made available to referees or only upon request.

- Confirmable Research. The main conclusions of the research can be attained independently without the use of software provided by the author. (But using the complete description of algorithms and methodology provided in the publication and any supplementary materials.)

- Auditable Research. Sufficient records (including data and software) have been archived so that the research can be defended later if necessary or differences between independent confirmations resolved. The archive might be private, as with traditional laboratory notebooks.

- Open or Reproducible Research. Auditable research made openly available. This comprised welldocumented and fully open code and data that are publicly available that would allow one to (a) fully audit the computational procedure, (b) replicate and also independently reproduce the results of the research, and (c) extend the results or apply the method to new problems.

*Source: V. Stodden, D.H. Bailey, J. Borwein, R.J. Leveque, W. Rider, and W. Stein (2014), Setting the Default to Reproducible Reproducibility in Computational and Experimental Mathematics

\section{Reproducibility Guidelines}

To address the reproducibility crisis, many stakeholders, including funders, journals, scientific societies, institutions, and individual researchers, have developed reproducibility guidelines and recommendations. Many of these guidelines outline measures that academic librarians are well positioned to support. This study examines a cross-section of these guidelines, specifically, the Transparency and Openness Promotion (TOP) Guidelines, the American Statistical Association (ASA) guidelines, the National Science Foundation's (NSF) guidelines, and the National Institutes of Health (NIH) guidelines. These guidelines were selected because of their applicability to many researchers, their broad applicability across disciplines, and the representative nature of the recommendations they contain. The measures in these guidelines will guide the discussion in the next section on how library services can support reproducible research. 
The Transparency and Openness Promotion (TOP) Guidelines are a set of eight standards for academic journals created in 2014 by the Center for Open Science (COS), journal editors, funding agency representatives, and disciplinary experts. ${ }^{17}$ The TOP Guidelines are a flexible set of standards that can be implemented at three levels depending on disciplinary norms and the ability to implement change. The first level requires disclosure of whether the standard has been followed (for example, a statement declaring whether data have been shared); the second level requires that the standard has been followed (for example, that data are actually shared); finally, the third level requires that the journal verifies that the standard has been followed.

This modular framework recognizes that there are different disciplinary norms for transparency and abilities to implement change across disciplines. In September 2017, Elsevier became a signatory to the TOP Guidelines for approximately 1,800 journals. ${ }^{18}$ As of April 2018, almost 5,000 journals and societies have signed on to the TOP Guidelines. ${ }^{19}$ As stated by the authors previously, the TOP Guidelines contain many recommendations academic librarians will find familiar (table 2). ${ }^{20}$

\section{TABLE 2 TOP Guidelines ${ }^{21}$}

- Citation: Proper citing of data, code, and materials and the recognition of these products as legitimate intellectual contributions to science. (Standard 1)

- Data Transparency, Analytic Methods (code) Transparency, Research Materials Transparency: These three distinct standards relate to the degree to which data, code, and research materials are made available to other researchers to enable reproducibility and replication. (Standards $\mathbf{2}, \mathbf{3}, \mathbf{4}$ )

- Design and Analysis Transparency: This standard encourages authors to follow explicit guidelines for disclosing key aspects of research design and analysis. For example, the PRISMA guidelines outline explicit standards for reporting systematic review research, and the ARRIVE Guidelines outline similar standards for reporting animal research. (Standard 5)

- Preregistration of Studies, Preregistration of Analysis Plans: Preregistration of studies involves publicly declaring the research you are conducting in advance and therefore increases discovery of research that was not published (thus addressing publication bias). Preregistration of analysis plans goes a step further by including details about planned analysis, preventing problems like $p$-hacking as well as certifying the distinction between confirmatory and exploratory research. (Standards $\mathbf{6 , 7 )}$

- Replication: This standard relates to the journal's willingness to publish direct replications of studies it previously published. (Standard 8)

Another set of guidelines, developed by the American Statistical Association (ASA), is aimed at influencing research funders. ${ }^{22}$ The ASA Guidelines also contain a number of "principles and observations" in the preamble that are more general and may be especially interesting to academic librarians. These principles and observations recognize the importance of open, citable, data, and code published in open repositories, as well as using computational practices during data analysis and processing. ${ }^{23}$

The ASA's recommendations include the following: ${ }^{24}$

- Funding small-scale software development, data products, and replications of previous studies.

- Increasing support for the methodological training, with particular emphasis on the need for data management skills.

- Adding code management plans to existing Data Management Plans (DMPs) and asking grant reviewers to explicitly assess DMPs. 
- Creating mandatory undergraduate reproducibility and computational research classes.

- Increasing the impact that robust and reliable research practices have on a researcher's chance of getting grants.

- Providing increased guidance and including more statisticians as part of grant review committees, and the development of standard terminology around reproducibility.

National research funders have also begun to address reproducibility. The National Institute of Health (NIH) hosted a joint workshop in 2014 with the Nature Publishing Group, the journal Science, and journal editors representing the 30 journals where NIH-funded research was most frequently published. ${ }^{25}$ The resulting five recommendations contained in the Principles and Guidelines for Reporting Preclinical Research call for rigorous statistical analysis; transparency in reporting methods; data and materials sharing; consideration of refutations; and building best practices around materials transparency and sharing. ${ }^{26}$ The most actionable recommendation here in terms of existing library services and expertise is for data and code sharing and the use of machine-readable formats in supplementary information. ${ }^{27}$

In 2014, the Office for the Management and Budget (OMB) of the NSF released "A Framework for Ongoing and Future National Science Foundation Activities to Improve Reproducibility, Replicability, and Robustness in Funded Research." ${ }^{28}$ While not an official set of guidelines, this framework document reviewed "the substantial amount of activity underway and anticipated at the NSF in key areas related to ensuring reproducibility, replicability, and robustness in funded research." 29 The authors of the guidelines specifically note the importance of data sharing and curation, including "methods, protocols, original data, data reductions, and analysis protocols as appropriate." ${ }^{30}$ They also call for increased transparency; ensuring the use of robust models, instrumentation, and interpretations; and increased publication of negative findings.

The following year the NSF Directorate for Social, Behavioral, and Economic Sciences released their own set of nine recommendations. ${ }^{31}$ These recommendations included a call for more robust methodologies and reporting standards so that reports of funded research

\begin{tabular}{|l|l|l|l|l|}
\hline \multicolumn{5}{|c|}{ Commonalities Between Major Reproducibility Guidelines } \\
\hline & $\begin{array}{l}\text { Transparency } \\
\text { and Openness } \\
\text { Promotion (TOP) }\end{array}$ & $\begin{array}{l}\text { American Statistical } \\
\text { Association (ASA) }\end{array}$ & $\begin{array}{l}\text { National Science } \\
\text { Foundation } \\
\text { (NSF) }\end{array}$ & $\begin{array}{l}\text { National } \\
\text { Institute of } \\
\text { Health (NIH) }\end{array}$ \\
\hline $\begin{array}{l}\text { Data Transparency } \\
\text { (Sharing) }\end{array}$ & Yes & $\begin{array}{l}\text { Yes (+ assessment of } \\
\text { DMPs by funders) }\end{array}$ & Yes & Yes \\
\hline $\begin{array}{l}\text { Software Transparency } \\
\text { (Sharing) }\end{array}$ & Yes & Yes & Yes & Yes \\
\hline $\begin{array}{l}\text { Materials Transparency } \\
\text { (Sharing) }\end{array}$ & Yes & & & Yes \\
\hline Methods Transparency & Yes & $\begin{array}{l}\text { Yes (study + } \\
\text { analysis plans) }\end{array}$ & Yes & Yes \\
\hline $\begin{array}{l}\text { Preregistration } \\
\text { Yes }\end{array}$ & Yes (directly fund) & $\begin{array}{l}\text { Yes (+ publishing } \\
\text { negative results) }\end{array}$ & Yes \\
\hline $\begin{array}{l}\text { Supporting } \\
\text { Replications }\end{array}$ & $\begin{array}{l}\text { Computational } \\
\text { methods / Scripting } \\
\text { analysis }\end{array}$ & & \\
\hline $\begin{array}{l}\text { Best Practices for } \\
\text { Methodology and } \\
\text { Analysis }\end{array}$ & & & \\
\hline
\end{tabular}


would contain enough detail to "enable an independent researcher to reproduce the results of the original researcher." 32 Other recommendations included sponsoring research into how to evaluate replications, documenting questionable and suboptimal research practices, and forming a committee to monitor these issues and propose changes to the grant-making process.

There are other guidelines that could be used by librarians, especially liaison librarians and functional specialists working with researchers from specific disciplines. As identified previously by the authors, two examples include the Federation of American Societies for Experimental Biology recommendations as well as the Society for Neuroscience's "Research Practices for Scientific Rigor: A Resource for Discussion, Training, and Practice," which provides discussion points and recommendations for methodological issues, data analyses, and transparency. ${ }^{33}$ The authors are currently working on a resource to make disciplinary guidelines easier to find and use.

\section{Academic Libraries and Reproducibility}

Many of the recommendations contained in the reproducibility guidelines outlined in the previous section coincide with core areas of academic librarianship. This section provides specific recommendations for services and expertise that align with these guidelines. Where possible, examples are provided of areas where this is already being done. In most cases, these services and expertise are already provided by academic libraries; extending them to support reproducibility will require that libraries thoughtfully frame and articulate service and expertise as supporting reproducible research.

In "A Manifesto for Reproducible Science," Munafò and colleagues propose a number of measures to improve research reproducibility. ${ }^{34}$ These measures are grouped into five themes: Methods, Reporting and Dissemination, Reproducibility, Evaluation, and Incentives. ${ }^{35}$ These themes reinforce the trends seen in reproducibility guidelines and act as a summary for the major areas that efforts to support reproducible research need to address; in this paper these themes also serve as a framework for organizing the ways academic library services and expertise can support reproducible research. These measures are summarized in table 4 below, and each is discussed in further detail in the following sections. Each section outlines potential academic library services that impact research reproducibility and then provides examples where this is being done.

\section{Theme: Methods}

The methods theme encompasses measures that can be implemented during the study design stage of research. For example, Munafò et al. include measures aimed at protecting against cognitive biases and improving independent methodological support. ${ }^{36}$

While most libraries cannot systematically support measures aimed at improving study design and statistical analysis, academic libraries do have a role as a connector to other experts that can directly support these areas, such as statistical consulting and research computing services. Academic librarians typically have institution-wide roles that can be leveraged to build relationships and connect researchers with the support they require. Especially at large institutions, librarians are much more likely to be aware of these services than students or faculty.

Furthermore, with the growth of data-intensive and computational research, students and researchers need increased training and support for managing data and workflows. Librarians 


\begin{tabular}{|c|c|c|}
\hline \multicolumn{3}{|c|}{$\begin{array}{l}\text { TABLE } 4 \\
\text { Library Services Contributing to Reproducibility }\end{array}$} \\
\hline Theme & Intervention & Type of Support \\
\hline \multirow[t]{3}{*}{ Methods } & $\begin{array}{l}\text { Support for data-intensive } \\
\text { research methodologies; } \\
\text { automation of data processing } \\
\text { and analysis }\end{array}$ & $\begin{array}{l}\text { Supporting computational and data-intensive research } \\
\text { methodologies, bibliometrics, and GIS. Supporting } \\
\text { researchers building data processing and analysis pipelines } \\
\text { in standard computational tools such as } \mathrm{R} \text { and Python }\end{array}$ \\
\hline & Support for systematic reviews & $\begin{array}{l}\text { Supporting systematic reviews and extending systematic } \\
\text { review methodologies to new domains; providing support } \\
\text { for systematic reviews across the scientific process. }\end{array}$ \\
\hline & $\begin{array}{l}\text { Connector to methodological } \\
\text { and statistical support units }\end{array}$ & $\begin{array}{l}\text { Connecting researchers to statistical and methodological } \\
\text { expertise }\end{array}$ \\
\hline \multirow[t]{5}{*}{$\begin{array}{l}\text { Reporting and } \\
\text { Dissemination }\end{array}$} & $\begin{array}{l}\text { Finding and using reporting } \\
\text { Guidelines }\end{array}$ & $\begin{array}{l}\text { Helping researchers find and use guidelines and checklists } \\
\text { (e.g. PRISMA, etc.) to improve methods reporting }\end{array}$ \\
\hline & $\begin{array}{l}\text { Providing repositories for pre- } \\
\text { registrations }\end{array}$ & $\begin{array}{l}\text { Providing institutional repositories capable of supporting } \\
\text { pre-registrations }\end{array}$ \\
\hline & $\begin{array}{l}\text { Helping researchers understand } \\
\text { and find repositories for pre- } \\
\text { registration }\end{array}$ & $\begin{array}{l}\text { Helping researchers find appropriate repositories for pre- } \\
\text { registration and helping new researchers understand why } \\
\text { preregistration is important }\end{array}$ \\
\hline & Open access publisher & Providing open access journal publishing platforms \\
\hline & Encouraging replications & $\begin{array}{l}\text { Encouraging replications through support, programming } \\
\text { (e.g., poster session featuring replication studies of graduate } \\
\text { students), and institutional open-access publishing }\end{array}$ \\
\hline \multirow{3}{*}{$\begin{array}{l}\text { Reproducibility } \\
\text { (Encouraging } \\
\text { Transparency } \\
\text { and Open } \\
\text { Science) }\end{array}$} & Data curation & Curating research data before depositing into repositories \\
\hline & $\begin{array}{l}\text { Active research data } \\
\text { management }\end{array}$ & $\begin{array}{l}\text { Helping researchers manage their research data before } \\
\text { and during research, working with quality assurance } \\
\text { offices, and training new lab members }\end{array}$ \\
\hline & Data/Code/Methods Sharing & $\begin{array}{l}\text { Helping researchers find appropriate repositories for } \\
\text { sharing data, running institutional data repositories, and } \\
\text { helping define standards for citation and sharing }\end{array}$ \\
\hline \multirow{2}{*}{$\begin{array}{l}\text { Evaluation } \\
\text { (Diversifying } \\
\text { Peer Review) }\end{array}$} & Educating researchers & $\begin{array}{l}\text { Educating researchers about new forms of peer-review } \\
\text { and publication }\end{array}$ \\
\hline & Supporting preprints & $\begin{array}{l}\text { Helping researchers find appropriate venues for } \\
\text { depositing preprints, understanding journal guidelines } \\
\text { (e.g., Sherpa Romeo) regarding copyright, and } \\
\text { negotiating with journals }\end{array}$ \\
\hline \multirow{3}{*}{$\begin{array}{l}\text { Incentives } \\
\text { (Rewarding } \\
\text { Open and } \\
\text { Reproducible } \\
\text { Practices) }\end{array}$} & Citation standards & $\begin{array}{l}\text { Helping create citation standards for data, code, research } \\
\text { materials, etc. }\end{array}$ \\
\hline & Education & $\begin{array}{l}\text { Teaching faculty, researchers, and students about how } \\
\text { different citation metrics work and the costs and benefits } \\
\text { of each, as well as the longevity of scholar identity (e.g., } \\
\text { ORCID) }\end{array}$ \\
\hline & $\begin{array}{l}\text { Citation data provider to } \\
\text { Tenure and Promotion (T\&P) } \\
\text { Committees }\end{array}$ & $\begin{array}{l}\text { Providing citation data for data, code, software, and } \\
\text { materials to tenure and promotion committees and } \\
\text { advocating for changes to academic incentives. }\end{array}$ \\
\hline
\end{tabular}


working with the digital humanities, bibliometrics, and geographic information systems (GIS) have long supported data-intensive and computational methodologies. Academic libraries have been involved in various digital scholarship projects. ${ }^{37}$ One example is the Mapping Prejudice Project, which is a collaboration between the University of Minnesota and Augsburg University. ${ }^{38}$ In this example, staff from the Borchert Map Library have served on a team working to compile a database and accompanying visualizations of racial covenants in Minneapolis. ${ }^{39}$

There is now a need to extend support to other disciplines by supporting computational tools like R, Python, and Git. These tools can improve reproducibility by automating data processing and analysis, thus increasing consistency and allowing the underlying code to be audited and shared. With these methodologies comes a concurrent need for education around data and code management, versioning, and documentation. The ASA guidelines specifically note that "most students and faculty have little training in how to organize their data and software so that their analyses are reproducible." 40

In "Good Enough Practices in Scientific Computing," Wilson et al. recommend a number of measures to improve reproducibility, including good data management practices, code sharing, proper documentation, open licensing, project organization, and versioning, among other measures. ${ }^{41}$ Sandve et al. have similar recommendations for reproducible computational research, and similar recommendations also exist for cognitive neuroscience. ${ }^{42}$ An example of librarians supporting these measures is Shirley Zhao's two-day course on reproducibility that covered both tools and workflows. ${ }^{43}$

Librarians can also directly support reproducibility for some methodologies, such as systematic reviews. As Gore and Jones explain, many systematic review guidelines, including Cochrane and the Campbell Collaboration, explicitly recommend including a librarian as part of the research team. ${ }^{44}$ Evidence suggests that involving librarians improves the quality and reproducibility of systematic review. ${ }^{45}$ Koffel analyzed reported systematic review search strategies and found that "librarian involvement was strongly associated with the use of many recommended search methods and could improve the quality of the review, contributing to the replicability and robustness of meta-analytic findings." ${ }^{16}$ Koffel and Rethlefsen found that only 22 percent of systematic reviews they analyzed provided at least one reproducible search strategy, and only 13 percent provided reproducible strategies for all databases. ${ }^{47}$ Librarian involvement was found to significantly predict the inclusion of a reproducible strategy.

While librarians have supported systematic reviews in the health sciences for many years, this methodology is becoming more common in other disciplines (such as psychology and education). There have also been suggestions that systematic reviews should be adopted across the scientific process as one way of directly improving the reproducibility of research. ${ }^{48}$ Future research will be needed to analyze the role of librarians in supporting systematic reviews outside the health sciences, but services exist currently at Cornell and the University of Minnesota. ${ }^{49}$

\section{Theme: Reporting and Dissemination}

The reporting and dissemination theme refers to measures that can improve how the methodology of studies is reported. Examples include promoting study and analysis plan preregistration and improving the quality of methodological reporting. ${ }^{50}$

One measure encountered in multiple guidelines is for researchers to adhere to reporting guidelines and checklists to improve methods reporting so that research can be fully evaluated and reproduced. Reporting guidelines are checklists of items that must be reported about how 
research was carried out and differ based on study design. The EQUATOR (Enhancing the QUAlity and Transparency Of health Research) Network, an international initiative run from the Centre for Statistics in Medicine at the University of Oxford, promotes the use of reporting guidelines to improve published health research; as of April 2018, the EQUATOR website contained 398 reporting guidelines for both quantitative and qualitative study designs. ${ }^{51}$

Health science librarians have been helping researchers use guidelines like the Preferred Reporting Items for Systematic Reviews and Meta-Analyses (PRISMA) checklist while conducting systematic reviews for many years, and librarians can generally help educate researchers about the guidelines available for different methodologies. ${ }^{52}$ According to EQUATOR, "librarians are excellently positioned to raise awareness of the importance of complete, accurate and transparent reporting of research studies amongst the clinicians and researchers with whom they work." ${ }^{53}$ In 2017, the Librarian Network of EQUATOR launched two initiatives for supporting reproducibility via guidelines. ${ }^{54}$

Another measure that appears across multiple reproducibility guidelines is preregistration of studies and analysis plans. Preregistering a study involves publicly registering the intent of the study and is a guard against the file drawer problem, where studies, especially those with negative results, disappear from the record because they are never published, thus creating a bias in the literature. A well-known site for study preregistration is ClinicalTrials.gov, the government-run site where researchers register clinical trials, including the outcomes they will measure, and post summary results. Preregistering an analysis plan goes a step further by including the exact analysis that will be carried out, thus guarding against various questionable research practices, such as p-hacking, as well as certifying the difference between exploratory and confirmatory research.

Academic librarians can contribute to preregistration in a number of ways. First, librarians can educate researchers, especially new researchers, on the benefits of preregistration and tools that can be used for preregistration like disciplinary repositories and workflow tools like the Open Science Framework (OSF), which allows both managing the research process and preregistering study and analysis plans on a single platform. ${ }^{55}$ Librarians can also teach clinicians and other consumers of research to look for preregistrations as part of the critical appraisal process when evaluating research (for instance, during journal clubs as part of medical education).

Finally, academic libraries are increasingly providing platforms for open access journals, textbooks, and other content. Without the pressures to publish novel and high-impact studies, journals run on academic library publishing platforms can be free to publish replications and null findings. As librarians work with locally hosted journals, they should encourage them to adopt reproducibility guidelines such as TOP and other reporting standards and to accept replications when possible. Library publishing platforms can thus help drive reproducible and open science.

\section{Theme: Reproducibility}

The reproducibility theme refers to measures that would support the ability to verify, reproduce, or potentially replicate the results of research (for example, by sharing data, code, and materials in open repositories). Academic libraries have invested considerable time and resources in developing expertise and services around data curation and sharing, and this is perhaps one of the easiest areas for major research libraries to immediately support. A recent survey of Association of Research Libraries member institutions found that nearly two thirds (51 of 80 ) of respondents provide data curation services, while another 13 indicated services were in 
development. ${ }^{56}$ Attention to curation needs has also resulted in significant investment of staff resources, with a total of 293 staff in 49 reporting libraries involved in data curation activities. ${ }^{57}$

Librarians have also been involved in building and supporting tools to support code sharing. For example, the Reproducibility Librarian at New York University, Vicky Steeves, is involved in developing and supporting an open source tool called ReproZip, which was designed to help users overcome technical obstacles of reproducing computational research by creating containers that gather together the software dependencies needed to execute code. ${ }^{58}$ As Steeves et al. argue, advocating for tools like ReproZip provides a venue to expand beyond data management services into "holistic support for reproducible research practices."59

Academic libraries can also encourage data and code sharing through educational initiatives, running institutional repositories, supporting researchers depositing materials, and assisting researchers in finding appropriate disciplinary repositories. Librarians should also be involved in developing citation formats and systems for new areas: for example, the Oregon Health and Science University Libraries collaborated on the Resource Identification Initiative (RII), which developed a citation standard and platform for key resources used in experiments. ${ }^{60}$ This allows researchers to cite specific reagents and other materials so other researchers can build on and replicate their work.

Finally, an important area where librarians can contribute is helping researchers manage their data during the research process itself, not just during planning or depositing final data in repositories. In fact, the Center for Open Science Strategic Plan includes among its eight goals that "librarians apply curation and data management expertise throughout the research lifecycle, not just retrospectively." 61 This type of active research data management, which involves working with research data during the active phase of the research lifecycle, could have significant benefits for reproducibility. For example, librarians at the University of Minnesota, including one of the authors, have developed a short intervention that can be done during lab meetings that teaches aspects of research data management while giving participants pragmatic advice that can be applied immediately to the lab's current research data. ${ }^{62}$

\section{Theme: Evaluation}

The evaluation theme refers to measures that would impact the evaluation of research, such as changes to the peer review process. Munafò et al. specifically list diversifying peer review through preprints and postpublication peer review as potential changes that could improve research reproducibility. ${ }^{63}$ Librarians should maintain familiarity with tools like SHERPA RoMEO that allow scholars to search for journal stances on open access, preprints, postprints, and other factors that often contribute to the openness of research. ${ }^{64}$

Perhaps the most impactful measure librarians could adopt would be changing how they educate new researchers about scholarly communication and peer review to reflect the measures being adopted and recommended by others. For example, Registered Reports, a new form of publication, has been adopted by 99 journals as of April $2018 .{ }^{65} \mathrm{In}$ a registered report, peer review and provisional acceptance occurs on the basis of the hypothesis and methodology before data collection occurs. This shifts peer review from evaluating the results and how novel they are to their technical details. Morey et al. have recommended that reviewers withhold peer review unless authors adopt open practices, in part to improve reproducibility of research. ${ }^{66}$ Going further, Nosek, Spies, and Motyl have recommended discarding prepublication peer review entirely, thus making publication trivial and making review a 
purely evaluative process instead of whether a paper should be published. ${ }^{67}$ While the decisions about whether to adopt these measures will need to be made by scientists themselves, librarians can help educate them on the potential impacts of each and provide training and support for the ones that are adopted.

\section{Theme: Incentives}

The incentives theme contains measures that could affect the rewards and pressures researchers face and therefore the type of research they do and the research practices they follow. Francis Collins, the NIH Director, has called the role of incentives "the most vexed issue" in fixing the reproducibility crisis. ${ }^{68}$ Traditionally, the currency of science has been journal publications, particularly publications in high-impact journals. Journals, especially traditional journals, prefer to publish novel and positive research over negative or confirmatory research, and institutions reward researchers who publish in these high-impact journals with grants, promotion, and tenure. ${ }^{69}$ Evidence suggests this bias against negative and confirmatory research ultimately harms reproducibility and rigor in science. As Johnson notes, "less funding for validation and reproduction of results, and more importantly, less prestige in doing so... lowers the trust and confidence in the system as a whole, allowing room for more and more falsified experiments to be accepted with minor scrutiny."70

Measures that could change the incentives researchers face could therefore provide an opportunity to improve rigor and reproducibility and incentivize replications of previous research. Munafò et al. recommend changes that would reward open and reproducible practices, including using badges to certify reproducible and transparent practices, adopting registered reports, funding replication studies, and rewarding open science practices during hiring and promotion. ${ }^{71}$

To address reproducibility, academic librarians must be cognizant of the incentives of science and how they impact grants, tenure, and promotion opportunities. There are also ways academic libraries could positively impact incentives. Academic libraries select, license, and maintain many of the systems that provide scholarly metrics, teach the relative costs and benefits of different metrics to graduate students and researchers through workshops and guides, and provide these data to administrators for tenure and promotion committees. As academic culture changes and data, code, and alternative metrics become more accepted and citation formats are developed to track the use of these products, academic libraries could broaden the scope of the metrics librarians teach about and provide in order to include these new metrics.

\section{Discussion/Conclusion}

Academic libraries and librarians can directly impact research reproducibility: from recommending reporting guidelines, to coauthoring systematic reviews, to supporting the many facets of scholarly publishing, the recommendations contained in reproducibility guidelines align with many of the services and expertise that academic libraries already provide. Reproducibility also provides a strong rationale for many emerging library services like data curation and sharing and support and training for computational methods and data-intensive research.

Reproducibility gets to the core of scientific enterprise; academic librarians will therefore need to be mindful when defining our role and determining where and how it interfaces with disciplinary experts and other related experts such as statisticians. Developing broad knowledge about issues around reproducibility generally and disciplinary differences specifically 
will be a prerequisite for taking a leadership role without risking both an opportunity to positively impact our users and their good will. Simply adding "reproducibility" to existing job or service descriptions will not be enough.

Supporting reproducible research includes broad roles for liaisons, functional specialists, and service providers; taking a leadership role within the academy will depend on providing professional development opportunities about reproducibility and how it interfaces with academic library practice. Educational initiatives that define major terms, explore disciplinary differences, and highlight existing tools and resources should be provided by both professional organizations and institutions. Guidelines are a particularly good place to start investigating how disciplines and funders view reproducibility. In some cases, librarians may need to develop more specific technical skills such as how to use workflow technologies like Open Science Framework and computational tools like $\mathrm{R}$ if they are to have an impact on data-intensive and computational methodologies. Systematic review searching is a similarly technical expertise that will need to be developed in disciplines outside health sciences.

Approaches to reproducibility will depend on the needs of the discipline, and liaison librarians may want to prioritize disciplinary-specific implications for certain aspects of reproducible research such as confirmatory research versus exploratory research and honest errors versus academic misconduct. Profiles exploring norms in various disciplines would be a beneficial addition to the academic library science literature. For example, a librarian who serves as the subject specialist for education or educational psychology could explore education-specific literature regarding reproducibility and replicability by Makel, Plucker, and others. ${ }^{72}$

In this article, the authors have attempted to provide a framework and set of exemplars for how academic libraries can support research by comparing current services and expertise to the recommendations contained in guidelines from funders and societies. The authors believe that this framework demonstrates that academic libraries have significant expertise and services to support research reproducibility. Indeed, in many areas, academic libraries are well placed to lead change in academic research institutions.

\section{Acknowledgements}

The authors acknowledge helpful and insightful feedback on an earlier version of this article from colleagues including Lisa Federer, Melissa Rethlefsen, and Jeffrey Spies.

\section{Notes}

1. Open Science Collaboration, "Estimating the Reproducibility of Psychological Science," Science 349 (2015).

2. Keith A. Baggerly and Kevin R. Coombes, "Deriving Chemosensitivity from Cell Lines: Forensic Bioinformatics and Reproducible Research in High-Throughput Biology," Annals of Applied Statistics 3 (2009).

3. John P.A. Ioannidis, "Why Most Published Research Findings Are False," PLoS Medicine 2 (2005).

4. Rick O. Gilmore et al., "Progress toward Openness, Transparency, and Reproducibility in Cognitive Neuroscience," Annals of the New York Academy of Sciences 1396 (2017).

5. C. Glenn Begley and Lee M. Ellis, "Drug Development: Raise Standards for Preclinical Cancer Research," Nature 483 (2012).

6. Bruce C. Gibb, "Reproducibility," Nature Chemistry 6 (2014).

7. Rasmus E. Benestad et al., "Learning from Mistakes in Climate Research," Theoretical and Applied Climatology 126 (2016).

8. Zacharias Maniadis, Fabio Tufano, and John A. List, “To Replicate or Not to Replicate? Exploring Reproducibility in Economics through the Lens of a Model and a Pilot Study," Economic Journal 127, no. 605 (2017); Thomas Herndon, Michael Ash, and Robert Pollin, “Does High Public Debt Consistently Stifle Economic Growth? A 
Critique of Reinhart and Rogoff," Political Economy Research Institute Working Paper Series (2013).

9. Matthew C. Makel and Jonathan A. Plucker, "Facts Are More Important Than Novelty: Replication in the Education Sciences," Educational Researcher 43 (2014).

10. Franklin Sayre and Amy Riegelman, "The Reproducibility Crisis and Academic Libraries," College $\mathcal{E}$ Research Libraries 79, no. 1 (2018).

11. Ibid.

12. Ibid.

13. Kenneth Bollen et al., "Social, Behavioral, and Economic Sciences Perspectives on Robust and Reliable Science" (paper presented at the Report of the Subcommittee on Replicability in Science Advisory Committee to the National Science Foundation Directorate for Social, Behavioral, and Economic Sciences, 2015), 3.

14. Ibid., 4.

15. Jeffrey T. Leek and Leah R. Jager, "Is Most Published Research Really False?" Annual Review of Statistics and Its Application 4 (2017): 111.

16. Victoria Stodden et al., "Setting the Default to Reproducible Reproducibility in Computational and Experimental Mathematics," (2014), available online at http://stodden.net/icerm_report.pdf [accessed 23 January 2019].

17. B.A. Nosek et al., "Promoting an Open Research Culture," Science 348, no. 6242 (2015).

18. "Center for Open Science Announces Elsevier as New Signatory to Top Guidelines," available online at https://cos.io/about/news/centre-open-science-announces-elsevier-new-signatory-top-guidelines/. [accessed 30 September 2017].

19. “Top Guidelines, " available online at https://cos.io/our-services/top-guidelines/ [accessed 9 April 2018].

20. Center for Open Science, "Guidelines for Transparency and Openness Promotion (Top) in Journal Policies and Practices" (paper presented at the COS, 2015).

21. Sayre and Riegelman, "The Reproducibility Crisis and Academic Libraries."

22. Karl Broman et al., "Recommendations to Funding Agencies for Supporting Reproducible Research" (paper presented at the American Statistical Association, 2017), 3-4.

23. Ibid.

24. Ibid.

25. NIH, "Principles and Guidelines for Reporting Preclinical Research," National Institute of Health (2014).

26. Ibid.

27. Ibid.

28. "A Framework for Ongoing and Future National Science Foundation Activities to Improve Reproducibility, Replicability, and Robustness in Funded Research" (2014), available online at https://www.nsf.gov/attachments/134722/public/Reproducibility_NSFPlanforOMB_Dec31_2014.pdf [accessed 25 May 2017].

29. Ibid., 1 .

30. Ibid., 2.

31. Bollen et al., "Social, Behavioral, and Economic Sciences Perspectives on Robust and Reliable Science."

32. Ibid., 5 .

33. Federation of American Societies for Experimental Biology, "Enhancing Research Reproducibility: Recommendations from the Federation of American Societies for Experimental Biology," 4 (2016), available online at http://www.faseb.org/Portals/2/PDFs/opa/2016/FASEB_Enhancing Research Reproducibility.pdf [accessed 11 January 2019]; Society for Neuroscience, "Research Practices for Scientific Rigor: A Resource for Discussion, Training, and Practice," (2015), available online at https://www.sfn.org/Advocacy/Policy-Positions/ResearchPractices-for-Scientific-Rigor [accessed 11 January 2019].

34. Marcus R. Munafò et al., "A Manifesto for Reproducible Science," Nature Human Behaviour 1 (2017).

35. Ibid.

36. Ibid.

37. Rikk Mulligan, Supporting Digital Scholarship. SPEC Kit 350. Washington, DC: Association of Research Libraries, May 2016, doi:10.29242/spec.350.

38. "About Us," available online at https://www.mappingprejudice.org/about-us/ [accessed 31 October 2017].

39. Kirsten Delegard and Kevin Ehrman-Solberg, "Playground of the People? Mapping Racial Covenants in Twentieth-Century Minneapolis," Open Rivers: Rethinking the Mississippi, no. 6 (2017).

40. Broman et al., "Recommendations to Funding Agencies for Supporting Reproducible Research," 3. (2017).

41. Greg Wilson et al., "Good Enough Practices in Scientific Computing," PLoS Computational Biology 113

42. Geir Kjetil Sandve et al., “Ten Simple Rules for Reproducible Computational Research,” (2013), available online at https://www.mn.uio.no/ifi/english/research/networks/clsi/earlier_seminars/2013/110913.html [accessed 
11 January 2019].

43. Shirley Zhao, "Principles and Practices for Reproducible Science," available online at https:/github.com/ shirl0207/reproducible_science [accessed 6 October 2017].

44. Genevieve C. Gore and Julie Jones, "Systematic Reviews and Librarians: A Primer for Managers," Partnership: The Canadian Journal of Library and Information Practice and Research 10 (2015).

45. Jonathan B. Koffel, "Use of Recommended Search Strategies in Systematic Reviews and the Impact of Librarian Involvement: A Cross-Sectional Survey of Recent Authors," PLoS ONE 10 (2015); Melissa L. Rethlefsen et al., "Librarian Co-Authors Correlated with Higher Quality Reported Search Strategies in General Internal Medicine Systematic Reviews," Journal of Clinical Epidemiology 68 (2015).

46. Koffel, "Use of Recommended Search Strategies in Systematic Reviews and the Impact of Librarian Involvement," 10.

47. Jonathan B. Koffel and Melissa L. Rethlefsen, "Reproducibility of Search Strategies Is Poor in Systematic Reviews Published in High-Impact Pediatrics, Cardiology and Surgery Journals: A Cross-Sectional Study," PLoS ONE 11 (2016).

48. Kristina A. Thayer et al., "Intersection of Systematic Review Methodology with the NIH Reproducibility Initiative," Environmental Health Perspectives 122 (2014).

49. "Cornell University Library Systematic Review Service," available online at https://www.library.cornell. edu/services/systematic-review [accessed 23 January 2019]; "Systematic Review Service Pilot," available online at https://www.lib.umn.edu/researchsupport/systematic-review-service [accessed 23 January 2019].

50. Munafò et al., "A Manifesto for Reproducible Science."

51. "Equator Network," available online at www.equator-network.org/ [accessed 12 April 2018].

52. "PRISMA: Transparent Reporting of Systematic Reviews and Meta-Analyses," available online at www. prisma-statement.org/ [accessed 9 April 2018].

53. Shona Kirtley, "Impactful Librarians: Identifying Opportunities to Increase Your Impact," Journal of EAHIL 11 (2015): 24.

54. "Librarian Action Plan," available online at www.equator-network.org/wp-content/uploads/2013/06/ Librarian-Action-Plan-Simple-Ideas.pdf [accessed 18 December 2017].

55. Jeffrey R Spies, "The Open Science Framework: Improving Science by Making It Open and Accessible," (dissertation, University of Virginia, 2013), doi:10.31237/osf.io/t23za.

56. Cynthia Hudson-Vitale et al., Data Curation. SPEC Kit 354. Washington, DC: Association of Research Libraries, May 2017, doi:10.29242/spec.354.

57. Ibid.

58. Vicky Steeves, Fernando Chirigati, and Rémi Rampin, “Using Reprozip for Reproducibility and Library Services," OSF, March 5, 2018, available online at https://osf.io/8z73c/ [accessed 11 January 2019].

59. Ibid., 9.

60. Anita Bandrowski et al., "The Resource Identification Initiative: A Cultural Shift in Publishing," F1000Research 4 (2015).

61. Center for Open Science, "Strategic Plan 2017-2020," available online at doi:10.31219/osf.io/x2w9h [accessed 18 December 2017].

62. Caitlin J. Bakker, "Data Management in the Lab," in The Medical Library Association Guide to Data Management for Librarians, ed. Lisa Federer (Lanham, MD: Rowman \& Littlefield Publishers, 2016).

63. Munafò et al., "A Manifesto for Reproducible Science."

64. "Sherpa/Romeo," available online at www.sherpa.ac.uk/romeo/index.php [accessed 18 December 2017].

65. "Registered Reports," Center for Open Science, available online at https://cos.io/rr/ [accessed 9 April 2018].

66. Richard D. Morey et al., "The Peer Reviewers' Openness Initiative: Incentivizing Open Research Practices through Peer Review, Royal Society Open Science 3 (2016).

67. Brian A. Nosek, Jeffrey R. Spies, and Matt Motyl, "Scientific Utopia," Perspectives on Psychological Science 7 (2012).

68. Francis S. Collins and Lawrence A. Tabak, “NIH Plans to Enhance Reproducibility," Nature 505 (2014): 613.

69. Nosek, Spies, and Motyl, "Scientific Utopia."

70. Richard P. Johnson, "Consume, Reproduce, Extend and Connect: Sustaining Our Research Lifecycle," Bulletin of the Association for Information Science and Technology 43 (2017): 28.

71. Munafò et al., "A Manifesto for Reproducible Science."

72. Matthew C. Makel and Jonathan A. Plucker, "An Introduction to Replication Research in Gifted Education," Gifted Child Quarterly 59, no. 3 (2015); Matthew C. Makel et al., "Replication of Special Education Research," Remedial and Special Education 37, no. 4 (2016). 